International Journal of Bifurcation and Chaos, Vol. 11, No. 11 (2001) 2887-2894

(c) World Scientific Publishing Company

\title{
DEFECT DYNAMICS DURING A QUENCH IN A BÉNARD-MARANGONI CONVECTION SYSTEM
}

\author{
W. GONZÁLEZ-VIÑAS, S. CASADO, J. BURGUETE, \\ H. MANCINI and S. BOCCALETTI \\ Department of Physics and Applied Mathematics, \\ Universidad de Navarra, Irunlarrea s/n, 31080 Pamplona, Spain
}

Received November 6, 2000; Revised December 18, 2000

\begin{abstract}
We report experimental evidence of defect formation and dynamics in a symmetry breaking transition for a conduction-convection Bénard-Marangoni system. As opposite to the behavior of perfect patterns, defects appear to interact in a spatial region, responsible for the formation of bounded states that survive much longer than the characteristic time scales. The analysis of the transient defect dynamics allows to define this defect interaction region in the space, giving rise to penta-hepta-like defects on top of the hexagonal pattern. Other defect configurations are shown to disappear rapidly either through dislocations moving toward the boundaries or through dislocation-dislocation annihilation. This evidence suggests that the scaling law of defects in the final structure versus quench time might be investigated by analyzing the probability of two or more dislocations to appear in the same interaction region.
\end{abstract}

\section{Introduction}

In the last decades, one of the most studied subjects in nonlinear science has regarded the formation and competition of pattern in space extended systems [Cross \& Hohenberg, 1993]. A growing pattern is often associated with the presence of phase singularities, or topological defects. Point defects in a field in two space dimensions are structures such that the circulation of the phase gradient is a multiple of $2 \pi$ on any path surrounding them. The geometrical phase is not defined in the core of the defect [Landsberg, 1992] (thence the name of phase singularities, or topologically stable defect [Lega, 1989; Tolouse \& Kléman, 1976]).

The formation, statistical properties and dynamics of defects have been the object of many studies in the past [Coullet et al., 1987] and have been investigated experimentally in fluid dynamics [Ciliberto et al., 1990] and in nonlinear optics [Arecchi et al., 1991]. Namely, the role of defects in mediating turbulence in large aspect ratio hydrodynamical systems was investigated in fluid thermal convection [Ahlers \& Behringer, 1978; Ciliberto et al., 1990], in surface waves [Gollub \& Ramshankar, 1991] and in numerical and analytical treatments of partial differential equations [Kawasaki, 1984; Bodenschatz et al., 1988].

A topological defect may be produced in two different ways. One possibility is that the order parameter of a two-dimensional complex field has a point where its amplitude vanishes, thus inducing a singularity in its phase. An example of this kind of defects is a local dislocation in a pattern where the field displays a zero value in its amplitude and a discontinuity in its phase [Arecchi et al., 1991]. There are other cases, however, such as hexagonal patterns, where the formed structures arise as a collective arrangement of different components of the order parameter (generally with different amplitudes) that concur to the formation of the global field. In the case of hexagons, three modes oriented at $120^{\circ}$ to each other concur to the formation of the pattern and the most stable topological defect is 
realized in a spatial position where only the amplitudes of two modes vanish at once, while the global amplitude of the field is not vanishing. An example of this latter situation is the so called pentahepta structure in hexagonal fields [Ciliberto et al., 1990].

In recent papers it has been argued that defects played a crucial role in the phase transitions that took place in the universe cooling process after the Big-Bang, yielding to the apparition of topological defects, which are intended to be the origin of cosmological structures as galaxies [Kibble, 1976; Zurek, 1996]. The theoretical argument is based on the fact that, when considering a system in a state just below a symmetry breaking transition, and when approaching the bifurcation point with the control parameter, the correlation length of the system tries in general to adapt adiabatically to its stationary value. However, the slowing down near the critical point limits the change rate of the correlation length by selecting a maximum velocity in the propagation of perturbations in the system. As a result, when the correlation length cannot follow anymore the variations in the control parameter, it freezes, and sets up its initial value after the breaking symmetry transition. Consequently, the phases of the order parameter evolve in isolated regions connected by the point defects. This also imposes an initial density of defects in the structure. This process is expected to lead to a universal scaling law for the defect density in the appearing structure, which only depends upon the space dimension, topology, and dissipative character of the system [Zurek, 1996].

In order to confirm the above argument, many experiments have been carried out in nonequilibrium phase transitions systems. We here recall the initial experiments with superfluid helium [Hendry et al., 1994; Ruutu et al., 1996; Bäuerle, 1996] and with liquid crystals [Chuang et al., 1991; Bowick et al., 1994], followed by other recent evidences in systems where the symmetry breaking transition occurs between two stationary nonequilibrium states, offered with a nonlinear optical device [Ducci et al., 1999] and in a hydrodynamic convection system [Casado et al., 2001].

The experiment in a convection system by Casado et al. [2001] differs from the one in nonlinear optics by Ducci et al. [1999], insofar as defects appear in the former case on top of a hexagonal structure, whereas in the latter case they correspond to zeroes in the amplitude of the scalar global field. In this case, the scaling exponents are different with respect to the ones predicted by Zurek [1996], and one possible explanation for this difference can be based on the fact that the ideal stationary defects correspond to dislocations in two of the three components of the order parameter.

In this paper we aim to study more closely the formation of the defects and their interaction in a conduction-convection bifurcation experiment for a Bénard-Marangoni system. The considered bifurcation is a breaking symmetry one, the state below (above) the transition point being a homogeneous conduction state (a hexagonal pattern). This bifurcation was characterized by Schatz et al. [1995] to be a subcritical one. However, due to the small value of the subcriticality, the system is already frozen when the control parameter $\varepsilon$ reaches the value $\varepsilon_{\alpha}$, which is very small. As a consequence, the subcriticality is not relevant to the expected behavior of the system.

\section{Experimental Set-Up}

In order to observe the dynamics of defects during the quench we put a fluid layer $(350 \mathrm{cS}$ silicone oil) in a cylindrical container of polyoximethylene (diameter $\phi=137 \mathrm{~mm}$ ). Below the fluid there is a polished metallic plate electrically heated from below. The power delivered to the heater is controlled by a computer (Fig. 1).

The chosen fluid is transparent to the light and is in the Oberbeck-Boussinesq regime for the temperature values selected in the experiment. Furthermore, the particularly high Prandtl number of the silicone oil warrants the slaving of the velocity field to the temperature field. Additionally, the distance between the primary and secondary bifurcations in the control parameter is much larger than the changes performed during the experimental procedure. The used fluid layer depth $(1.9 \mathrm{~mm})$ induces a mostly surface tension driven instability.

We have measured the local temperatures by use of three T-type thermocouples (see Fig. 1), located in three different positions. Namely, the first one is located under the metallic plate $\left(T_{1}\right)$, the second one over the metallic plate $\left(T_{2}\right)$ and the third one on the fluid surface $\left(T_{3}\right)$. Furthermore, $T_{2}$ and $T_{3}$ were put in the cell center only when necessary for the measurement. All thermocouples are connected with a computer controlled multimeter, providing measurements on the local 


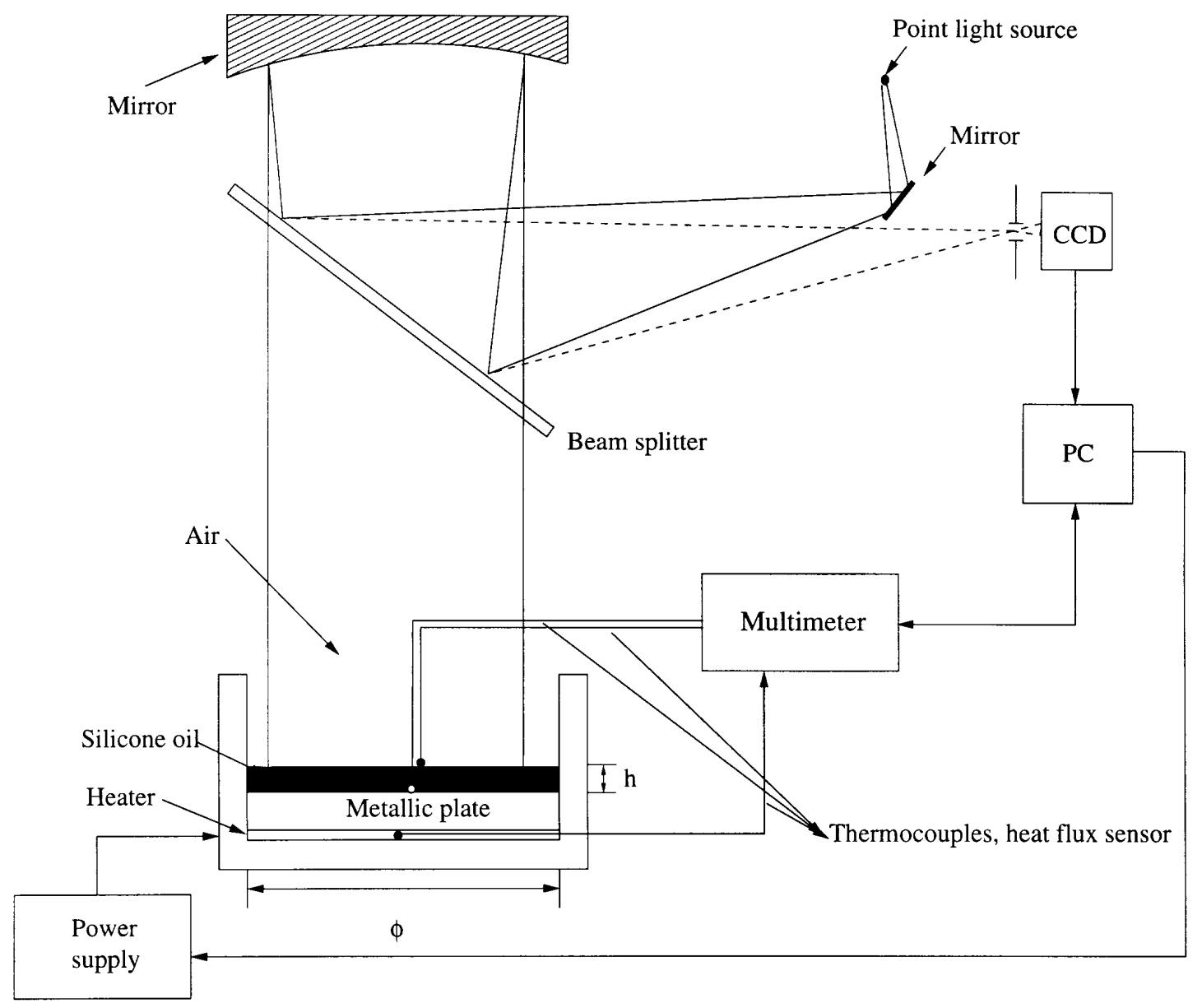

Fig. 1. The picture schematize the experimental setup and the shadowgraph technique used. The fluid layer depth is $h=1.9 \mathrm{~mm}$ and the diameter of the container is $\phi=137 \mathrm{~mm}$.

temperature fields. The information about the global temperature field is gathered independently through shadowgraph-type techniques [Mancini, 1994; Ondarçuhu et al., 1994], and the resulting images are captured by a CCD camera and sent to a computer.

In the following, we try to summarize the measurement trial. By initially applying a power $P_{1}$ to the heater, we set the system in the stationary conductive state (see Fig. 2, left) just below the convective threshold. In our case, the control parameter of the bifurcation is the reduced temperature difference, defined by $\varepsilon=\left(\Delta T-\Delta T_{c}\right) / \Delta T_{c}$. Here $\Delta T=T_{2}-T_{3}$ and $\Delta T_{c}$ corresponds to the value of $\Delta T$ for which the conduction state comes out to be unstable. After having prepared the system as above, we suddenly increase the power delivered to the system to $P_{2}$ (see curve in Fig. 2, up). As a result, $\varepsilon$ approaches asymptotically its new stationary value increasing, in the studied time range, approximately linearly at a rate of $2.3 \cdot 10^{-2} \mathrm{~min}^{-1}$. This rate depends on $P_{2}-P_{1}$ (see curve in Fig. 2, down).

During the quench, images are captured every fifteen seconds, ${ }^{1}$ forming a movie, and describing how the patterned structure forms. This way one obtains images (like the shadowgraph shown in Fig. 2, right) where bright zones mark to hot points with the fluid going upwards. Casado et al. [2001] proposes an analysis of these images, by associating them to the Voronoi cells [Voronoi, 1908] of the hot points, and by identifying the connectivity properties of each point (coordination number, etc.). Here, instead, we will present a complementary analysis of the measured patterned structures, based on complex demodulation techniques [Burguete, 1995; Kolodner \& Williams, 1990; Bloomfield, 1976], that we apply to an almost

\footnotetext{
${ }^{1}$ The vertical thermal diffusion time is around $32 \mathrm{~s}$.
} 


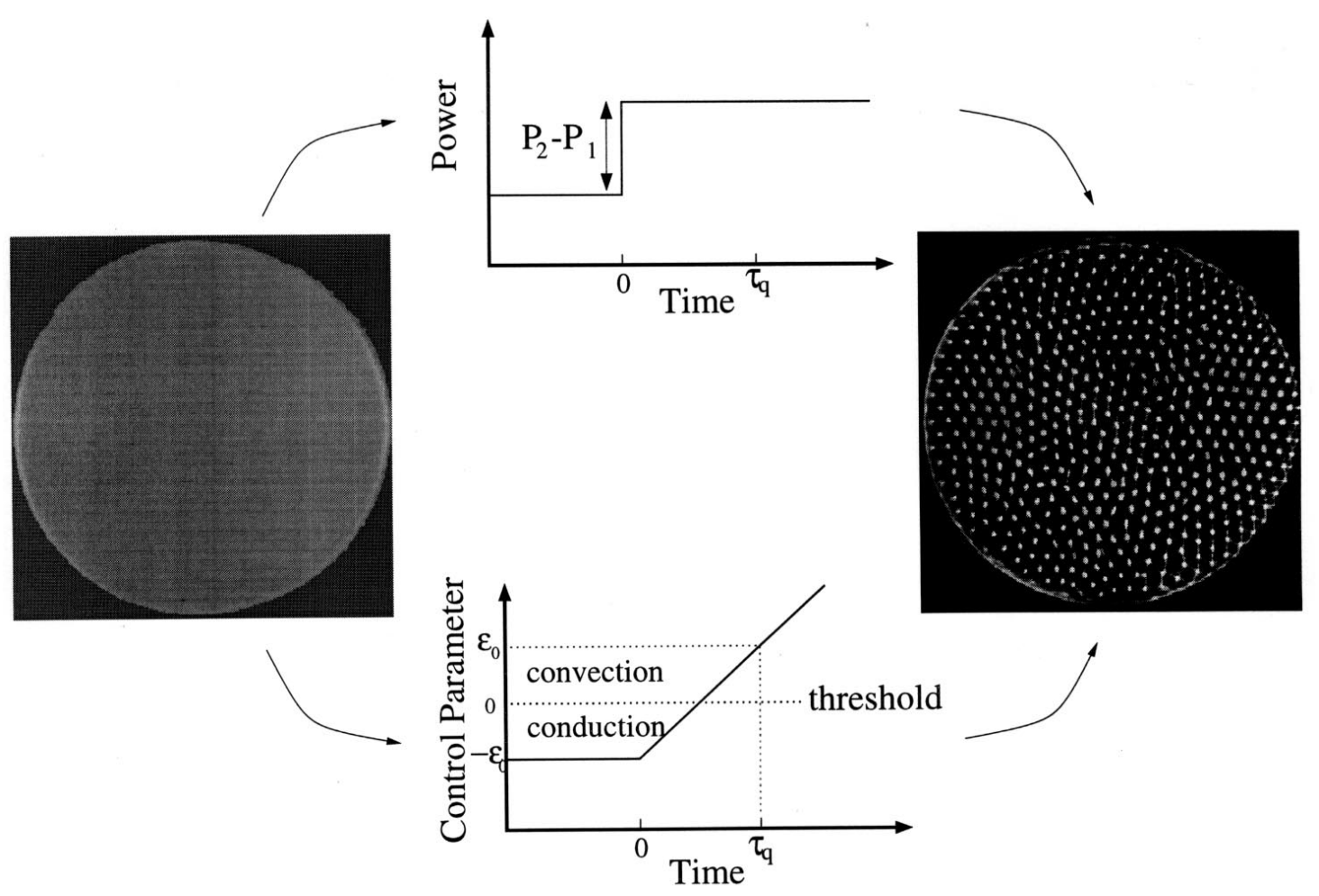

Fig. 2. Sketch of the experimental process. By starting with a conductive homogeneous steady state (left pattern) and by applying a step in the power delivered to the system (upper plot), we induce a linear increase in the control parameter (lower plot). As a consequence, the system crosses the conduction-convection threshold and reaches a fixed control parameter value after a time $\tau_{q}$. During the quench a movie (with images like the one shown in the right pattern) is taken in which the bright points correspond to the hotter fluid going upwards.

perfect region [Fig. 3(a)] (of size around seven wavelengths) ${ }^{2}$ of the last image of the movie (with few defects), as well as to the same region for all the previous images.

\section{Results and Discussion}

In our experiment, defects appear mainly associated to dislocations in one (or more) of the components of the order parameter. In the case in which dislocations occur simultaneously in more than one component, they can be either isolated or bounded to each other. As already discusses, a dislocation of a two-dimensional field is a point-like structure where the geometrical phase is not defined. As a consequence, the amplitude of the corresponding component must vanish in order to yield regularity in the whole order parameter. In general, defects in different modes strongly interact with each other, due to the nonlinear coupling between the corresponding system components. As a result of this interaction, one may be presented with defect bounded states. One possibility is a pair of near defects in the same component of the order parameter (usually two dislocations with opposite topological charge), whose spatial position evolve in the course of the time due to the intra-mode defect-defect interaction. Another possibility is having defects (not necessarily with opposite topological charge), each one affecting a different component of the order parameter, and evolving due to the inter-mode defectdefect interaction.

In a weakly nonlinear Bénard-Marangoni convection system, the underlying structure is an hexagonal pattern, realized by three different modal components. In a perfect hexagonal structure the most stable defect is realized by means of a pair of dislocations (with opposite topological charge and same spatial position), that occur in two of the three components of the order parameter, corresponding to the two independent geometrical phases of a hexagon [Echebarria, 1998; Lauzeral et al., 1993].

\footnotetext{
${ }^{2} \lambda$ being the wavelength, the aspect ratio $\phi / \lambda$ of the whole image is around 19.
} 
In this paper, we report the apparition of bounded (stable) defects corresponding to dislocations in two of the three components of the order parameter. Our first observation is that, contrary to what should have been expected, the positions of the two dislocations are not the same, as it can be seen from the complex demodulated phase of the three modes [Fig. 3(c)]. This could be related to finite size effects in the system or the presence of a dominant transient (and therefore nonlinear) state.

However, to better understand the above experimental evidence, one should consider the fact that a whole band of modes is in fact excited during the transition (the final control parameter is $\varepsilon \sim 0.1$ ), allowing a spatial modulation of the modes orienta- tion. In Fig. 3(b) we report the power spectrum of the chosen region, showing that a principal hexagonal structure is formed, which however is enlarged by the residual spatial dependence of the modal amplitudes. As a consequence, modes 2 and 3 have a domain-like Fourier transform, that corresponds to a whole set of near excited wavevectors realized either with the same wavenumber and different orientations or with same orientation, but slightly different wavenumbers. Experimentally, one observes that the corresponding domain-like regions in the real space have a very similar orientation, and it is possible to move along the global pattern from one to another of such regions in a continuous way, that is without discontinuities in the value of the local wavevectors. This is in agreement with a)

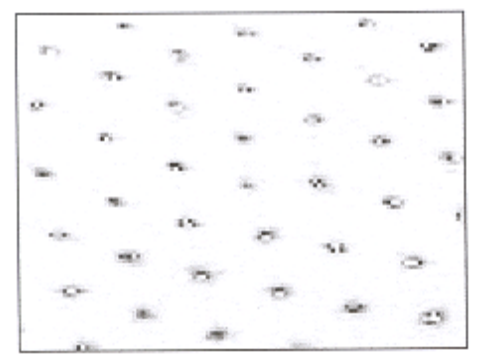

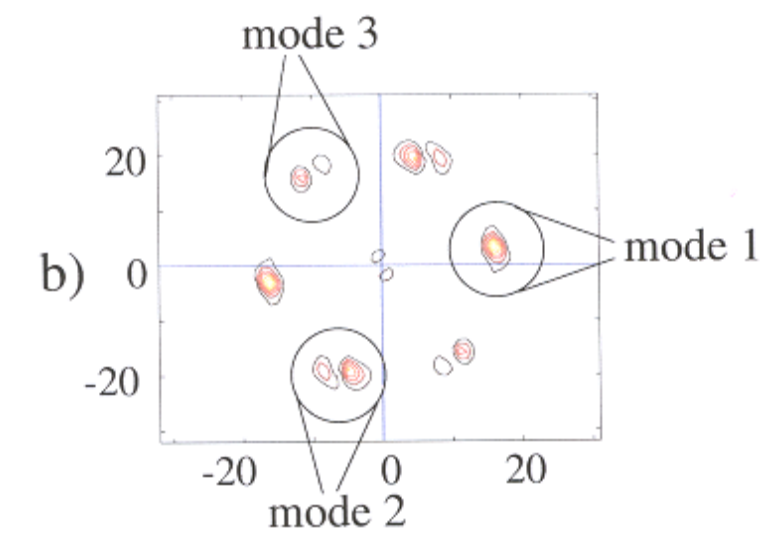

c)

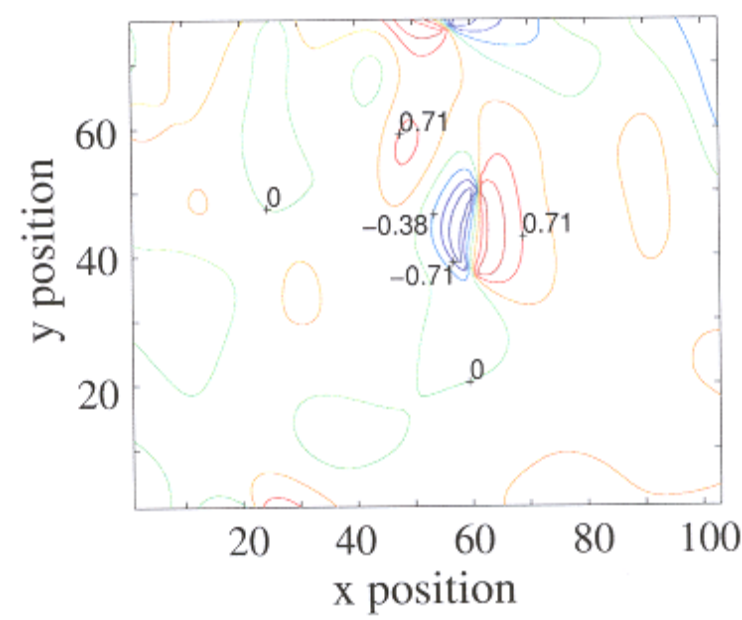

Fig. 3. Complex demodulation analysis for the intensity field of the studied region corresponding to the movie's last image. (a) Region of analysis (b) Modulus of its Fourier transform where the encircled regions identify the involved modes. (c) Contour plot for the synchronization parameter $F=-\cos \left(\phi_{1}+\phi_{2}+\phi_{3}\right)$ where $\phi_{j}$ are the phases of the modes originating the hexagonal pattern [Tsimring \& Rabinovich, 1995]. The singularities of the phases can be observed directly in the synchronization parameter as slightly displaced one from the other. 


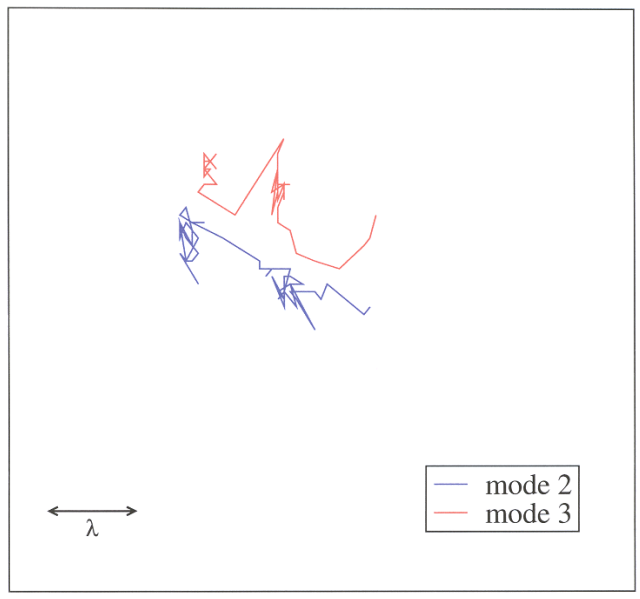

Fig. 4. This figure shows the trajectories of the defects found by complex demodulation of the images corresponding to the last 12 minutes of the movie. It can be seen as a drift of a bound state along the low-angle grain boundary. Mode 2 presents gliding motion and mode 3 presents climbing motion. In the figure the wavelength is marked, and the dislocations shown move to the bottom-right side. the model of a low-angle grain boundary as a sequence of edge dislocations [Kittel, 1956]. However, here the sequence of dislocations belongs to different modes.

As a second step of our analysis, we investigate on the bounded state formed by the two dislocations. To this purpose, we follow the trajectories of the two dislocations as they move (Fig. 4). Both dislocations move along the domain-like boundary. While the results indicate that one dislocation (that of mode 2) moves approximately in the direction of the wavevector of the second component (glides), the other one (that of mode 3) moves perpendicularly to the wavevector of the third component (climbs). The relevant consequence is that one cannot refer to a defect position, but rather to a defect region wherein the interaction between the two dislocations takes place to form a bounded defectlike state. This region has a diameter of about

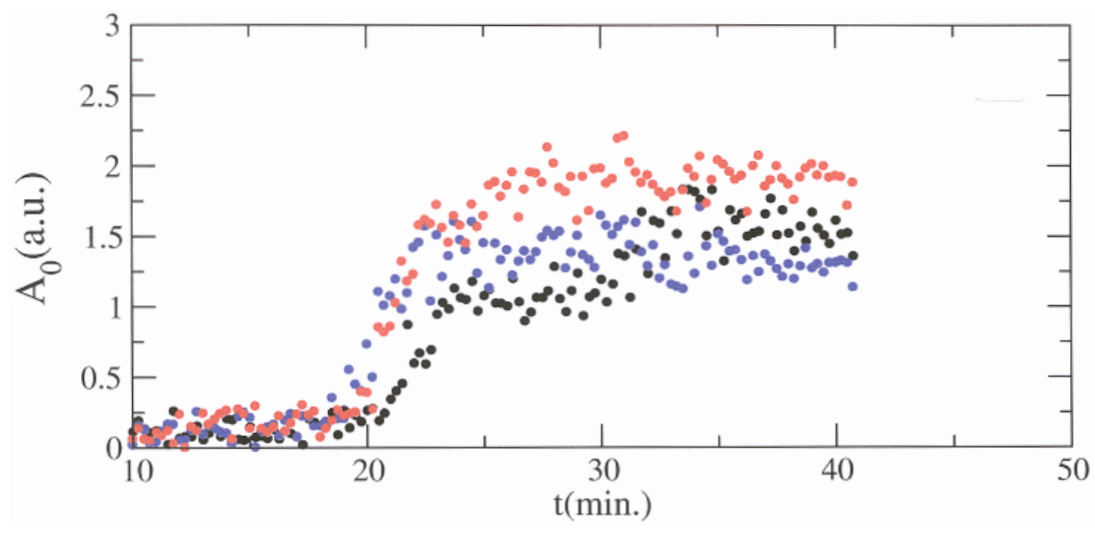

(a)

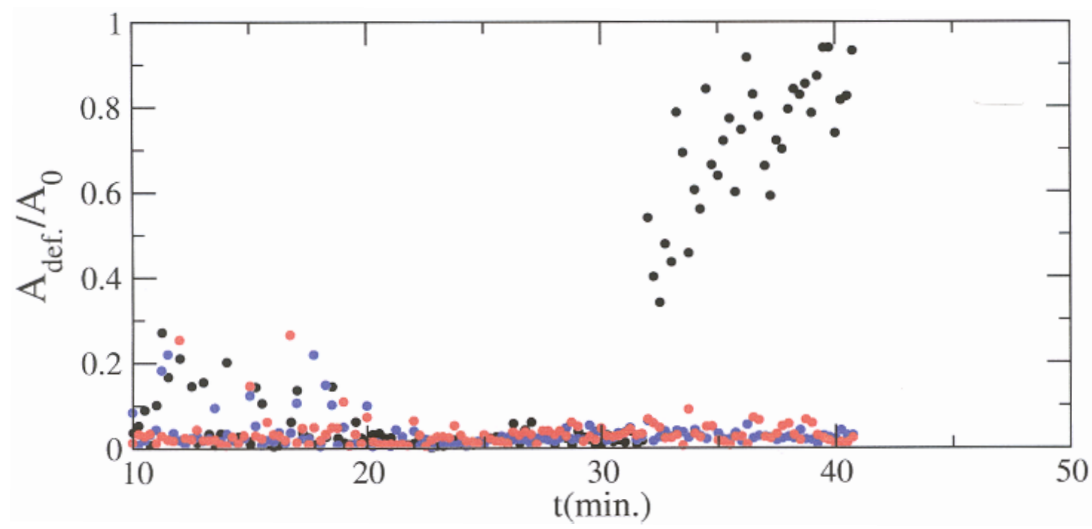

(b)

Fig. 5. This figure shows the amplitudes for each mode, obtained by complex demodulation as a function of time. (a) Amplitudes for each mode outside the defect interaction region. (b) Amplitudes of the defect, normalized by the amplitude of (a). 
two or three wavelengths. In the following, when speaking of amplitude of a defect, we mean the minimum amplitude of the considered mode within the defect region. Therefore, if a given mode does not show a dislocation within the defect region, then its amplitude will be appreciably different from zero.

In order to visualize what is happening during the pattern forming transition, we report the amplitudes of the three components far from the considered defect region [Fig. 5(a)] as well as the amplitudes of the modes within the defect region [Fig. 5(b), amplitudes normalized to the corresponding values of Fig. 5(a)] in the course of the time. Before the quench, the system is below threshold and all modes have zero amplitudes. After the quench, a hexagonal structure begins to form at $t \simeq 20 \mathrm{~min}$. [see Fig. 5(a)], that shows a simultaneous zero in the three amplitudes within the defect region [Fig. 5(b)]. Comparing Figs. 5(a) and Fig. 5(b), it is easy to notice that a penta-hepta-like defect is forming at $t \simeq 32 \mathrm{~min}$, due to a dislocation in the mode 1 exiting out of the defect region. However, such a defect forms a while after the global hexagonal structure has appeared. Although this seems to indicate that the defects in the final structure after the quench come from zeroes of the global field, in fact other dislocations have been observed near the threshold without their counterparts in the other modes.

In conclusion, our experimental results show that defects in a symmetry breaking transition appear randomly in space and in modes. The unstable configurations rapidly disappear through dislocations rapidly moving toward the boundaries or annihilating with other dislocations (with opposite topological charge) [Tsimring \& Rabinovich, 1995], leading to a final state where defects are present only in the form reported by Casado et al. [2001].

\section{Acknowledgments}

Work partly supported by the Spanish DGICYT contract n. PB98-0208. S. Casado acknowledges financial support from the "Asociación de Amigos de la Universidad de Navarra".

\section{References}

Ahlers, G. \& Behringer, R. P. [1978] "Evolution of turbulence from the Rayleigh-Bénard instability," Phys. Rev. Lett. 40(11), 712-716.
Arecchi, F. T., Giacomelli, G., Ramazza, P. L. \& Residori, S. [1991] "Vortices and defect statistics in two-dimensional optical chaos," Phys. Rev. Lett. 67(27), 3749-3752.

Bäuerle, C., Bunkov, Y. M., Fischer, S. N., Godfron, H. \& Pickett, G. R. [1996] "Laboratory simulation of cosmic string formation in the early universe using superfluid ${ }^{3} \mathrm{He}, "$ Nature 382, 332-334.

Bloomfield, P. [1976] Fourier Analysis of Time Series: An Introduction (Wiley, NY).

Bodenschatz, E., Pesch, W. \& Kramer, L. [1988] "Structure and dynamics of dislocations in anisotropic pattern-forming systems," Physica D32, 135-145.

Bowick, M. J., Chandar, L., Schiff, E. A. \& Srivastava, A. M. [1994] "The cosmological Kibble mechanism in the laboratory: String formation in liquid crystals," Science 263, 943-945.

Burguete, J. [1995] Inestabilidades Convectivas Producidas por un Calentamiento Localizado, $\mathrm{PhD}$ thesis, Universidad de Navarra. Pamplona, Spain.

Casado, S., González-Viñas, W., Mancini, H. \& Boccaletti, S. [2001] "Topological defects after a quench in a Bénard-Marangoni convection system," Phys. Rev. E63(5), 57301.

Chuang, I., Dürrer, R., Turok, N. \& Yurke, B. [1991] "Cosmology in the laboratory: Defect dynamics in liquid crystals," Science 251, 1336-1342.

Ciliberto, S., Coullet, P., Lega, J., Pampaloni, E. \& Pérez-García, C. [1990] "Defects in roll-hexagon competition," Phys. Rev. Lett. 65(19), 2370-2373.

Coullet, P., Elphick, C., Gil, L. \& Lega, J. [1987] "Topological defects of wave patterns," Phys. Rev. Lett. 59(8), 884-887.

Cross, M. C. \& Hohenberg, P. C. [1993] "Pattern formation outside of equilibrium," Rev. Mod. Phys. 65(3), 851-1112.

Ducci, S., Ramazza, P. L., González-Viñas, W. \& Arecchi, F. T. [1999] "Order parameter fragmentation after a symmetry-breaking transition," Phys. Rev. Lett. 83(25), 5210-5213.

Echebarria, B. [1998] Inestabilidades Termocapilares en una Capa de Fluido, $\mathrm{PhD}$ thesis, Universidad de Navarra, Pamplona, Spain.

Gollub, J. P. \& Ramshankar, R. [1991] "Spatiotemporal chaos in interfacial waves," in New Perspectives in Turbulence, ed. Sirovich, L. (Springer-Verlag, NY), pp. $165-172$.

Hendry, P. C., Lawson, N. S., Lee, R. A. M., McClintock, P. V. E. \& Williams, C. D. H. [1994] "Generation of defects in superfluid ${ }^{4} \mathrm{He}$ as an analogue of the formation of cosmic strings," Nature 368, 315-317.

Kawasaki, K. [1984] "Topological defects and nonequilibrium," Prog. Theor. Phys. Suppl. 79, 161-180.

Kibble, T. W. B. [1976] "Topology of cosmic domains and strings," J. Phys. A9(8), 1387-1398.

Kittel, C. [1956] Introduction to Solid State Physics, 2nd 
edition, Wiley Series on the Science and Technology of Materials (John Wiley, NY).

Kolodner, P. \& Williams, H. [1990] "Complex demodulation techniques for experiments on travelingwave convection," in Nonlinear Evolution of SpatioTemporal Structures in Dissipative Continuous Systems, eds. Busse, F. H. \& Kramer, L. (Plenum Press, NY), pp. 73-91.

Landsberg, A. S. [1992] "Geometrical phases and symmetries in dissipative systems," Phys. Rev. Lett. 69(6), $865-868$.

Lauzeral, J., Metens, S. \& Walgraef, D. [1993] "On the phase dynamics of hexagonal patterns," Europhys. Lett. 24, 707-712.

Lega, J. [1989] Défauts Topologiques Associés à la Brisure de l'Invariance de Translation dans le Temps, $\mathrm{PhD}$ thesis, Université de Nice, Nice, France.

Mancini, H. L. [1994] Osciladores Térmicos de BénardMarangoni, PhD thesis, Universidad de Navarra, Pamplona, Spain.

Ondarçuhu, T., Mindlin, G., Mancini, H. L. \& PérezGarcía, C. [1994] "The chaotic evolution of patterns in Bénard-Marangoni convection with square symmetry," J. Phys.: Condens. Matter 6, A427-A432.
Ruutu, V. M. H., Eltsov, V. B., Gill, A. J., Kibble, T. W. B., Krusius, M., Makhlin, Y. G., Placais, B., Volovik, G. E. \& Xu, W. [1996] "Vortex formation in neutronirradiated superfluid ${ }^{3} \mathrm{He}$ as an analogue of cosmological defect formation," Nature 382, 334-336.

Schatz, M. F., VanHook, S. J., McCormick, W. D., Swift, J. B. \& Swinney, H. L. [1995] "Onset of surfacetension-driven Bénard convection," Phys. Rev. Lett. 75(10), 1938-1941.

Tolouse, G. \& Kléman, M. [1976] "Principles of a classification of defects in ordered media," J. Phys. Lett. 37, 149-180.

Tsimring, L. S. \& Rabinovich, M. I. [1995] "Dislocations in hexagonal patterns," in Spatio-Temporal Patterns in Nonequilibrium Complex Systems, eds. Cladis, P. E. \& Palffy-Muhoray, P., Santa Fe Institute (Addison-Wesley), pp. 295-304.

Voronoi, G. [1908] "Nouvelles applications des parametres continus a la theorie des forms quadratiques. duesieme memoire: Recherches sur les paralleloderes primitifs paralleloderes primitifs," J. Reine Angew. Math. 134, 198-287.

Zurek, W. H. [1996] "Cosmological experiments in condensed matter systems," Phys. Rep. 276(4), 177-221. 